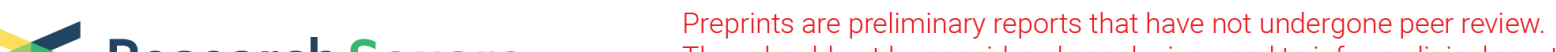 $\begin{array}{ll}\text { Research Square } & \text { They should not be considered conclusive, used to inform clinical practice, } \\ \text { or referenced by the media as validated information. }\end{array}$
}

\section{Microbiome composition comparison in oral and atherosclerotic plaque from patients with and without periodontitis}

\section{Daichi Isoshima}

Okayama University Graduate School of Medicine, Dentistry, and Pharmaceutical Sciences

Keisuke Yamashiro ( $\nabla$ yamash-k@cc.okayama-u.ac.jp)

Okayama University Hospital https://orcid.org/0000-0003-1208-892X

\section{Kazuyuki Matsunaga}

Brain Attack Center Ota Memorial Hospital

Makoto Taniguchi

Taniguchi Dental Clinic

\section{Takehiro Matsubara}

Okayama University Graduate School of Medicine, Dentistry, and Pharmaceutical Sciences

Shuta Tomida

Okayama Graduate School of Medicine, Dentistry, and Pharmaceutical Sciences

\section{Shinzo Ota}

Brain Attack Center Ota Memorial Hospital

\section{Michiyoshi Sato}

Brain Attack Center Ota Memorial Hospital

\section{Yutaka Shimoe}

Brain Attack Center Ota Memorial Hospital

\section{Tatsuo Kohriyama}

Brain Attack Center Ota Memorial Hospital

\section{Arias Zulema}

Okayama University Graduate School of Medicine, Dentistry, and Pharmaceutical Sciences

\section{Kazuhiro Omori}

Okayama University Hospital

\section{Tadashi Yamamoto}

Okayama University Graduate School of Medicine, Dentistry, and Pharmaceutical Sciences

\section{Shogo Takashiba}

Okayama University Graduate School of Medicine, Dentistry, and Pharmaceutical Sciences 
Keywords: microbiome; atherosclerosis; periodontitis; next-generating sequencing; oral bacteria

Posted Date: August 2nd, 2019

DOI: https://doi.org/10.21203/rs.2.12329/v1

License: (c) (1) This work is licensed under a Creative Commons Attribution 4.0 International License. Read Full License

Version of Record: A version of this preprint was published at Odontology on May 19th, 2020. See the published version at https://doi.org/10.1007/s10266-020-00524-w. 


\section{Abstract}

Background There is no conclusive evidence regarding a causal relationship between periodontitis and atherosclerosis. In this study, we examined the microbiome in the oral cavity and atheromatous plaques from atherosclerosis patients with or without periodontitis to investigate the role of oral bacteria in the formation of atheromatous plaques. We chose four patients with and without periodontitis, who had undergone carotid endarterectomy. Bacterial samples were extracted from saliva on the tongue surface, from periodontal pocket (during the oral examination), and from the atheromatous plaques. We investigated the general and oral conditions from each patient and performed next-generation sequencing analysis for all bacterial samples. Results There were no significant differences between both groups concerning general conditions. However, the microbiome patterns of the gingival pocket showed differences depending on the absence or presence of periodontitis, while those of the saliva were relatively similar. The microbiome pattern of the atheromatous plaques was entirely different from that in saliva present on the tongue surface and gingival pocket, and oral bacteria were seldom detected. However, the microbiome pattern in atheromatous plaques was different in the presence or absence of periodontitis. Conclusion These results indicated that oral bacteria did not affect the formation of atheromatous plaques directly. However, the metabolic products of microbiome or the host inflammatory response might indirectly influence the composition of atheromatous plaques.

\section{Introduction}

Periodontitis is a predominant oral infectious disease in which an excessive immune response directed at the microbiome on the tooth surface destroys the periodontal tissue, forming periodontal pockets. The microbiome in these pockets includes pathogenic anaerobic bacteria that can form biofilms, which are inherently more resistant to antibacterial compounds and host immune components [1]. The mature biofilm causes further periodontitis progression because of the prolonged inflammation associated with the protracted immune response. Therefore, periodontitis has two main features: it is an infectious disease caused by microbiome imbalance and a chronic inflammatory disease caused by a dysregulated immune response.

These two characteristic features of periodontitis are shared by various systematic diseases, including diabetes, arteriosclerosis, cardiovascular diseases such as stroke and infective endocarditis, brain diseases, cancer, and non-alcoholic steatohepatitis, and increase the risks of preterm birth and low birth weight [2-9]. Arteriosclerosis includes atherosclerosis, in which an atherosclerotic plaque is formed on the blood vessel walls [10]. Briefly, the mechanism of plaque formation involves an endothelial dysfunction with various causes, such as hypertension, diabetes, obesity, and high cholesterol levels. All feature the accumulation of low-density lipoprotein ( $L D L)$ cholesterol between the intimal and medial endothelial surfaces. Monocytes that differentiate into macrophages ingest oxidized LDL cholesterol and die. Subsequently the cholesterol and lipid composition form an atherosclerotic plaque [11]. An association between atherosclerosis and periodontitis has been suggested in some epidemiology reports. Moreover, bacterial investigations aimed at detecting periodontal bacteria in the atherosclerotic plaque have been 
conducted [12-14]. Although observational data support an association between periodontitis and atherosclerotic vascular disease, the data do not yet justify a causative relationship [15]. Multiple common factors that include diabetes, high blood pressure, dyslipidemia, and smoking habits affect the disease progression. However, there is little data concerning the direct involvement of periodontal bacteria in the development of atherosclerotic vascular disease $[16,17]$.

Porphyromonas gingivalis $(P g)$ is the most prominent pathogen in periodontal disease. Many studied have addressed the association between $P g$ and atherosclerotic vascular disease in clinical and animal models. Some clinical analyses sought to detect the DNA of $P g$ in atherosclerotic plaques [18-21]. We also reported the significant correlation of plasma IgG titer to $P g$, -which are higher in patients with severe periodontitis, with the level of LDL cholesterol [22]. Furthermore, in a mice model, an association between periodontitis and atherosclerotic vascular disease was demonstrated using $P g$ [23]. Ford et al. [24] also reported that intraperitoneal immunizations with $P g$ enhance levels of serum antibodies to $P g$ and atherosclerosis. Hypercholesterolemia and subsequent arteriosclerosis occurs in apolipoprotein $\mathrm{E}$ knockout mice $\left(\mathrm{ApoE}^{-/}\right)$, which have been used to investigate the association between periodontitis and atherosclerosis. Lalla et al. [2] reported that oral infection with $P g$ accelerates early atherosclerosis in $\mathrm{ApoE}^{-/-}$mice. Maekawa et al. [25] reported that chronic oral infection with $P g$ accelerates atheroma formation in ApoE ${ }^{\text {shl }}$ mice. However, the authors did not detect Pg DNA (16S-ribosomal RNA) by PCR in the aortic valve. Thus, how $P g$ induce atheroma formation is still unknown. The impact of the disruption of the normal microbiome on various diseases is unclear and a comprehensive analysis is necessary, since the microbiome could contribute to the formation of the atheroma microbiome.

Microbiome analysis has typically involved bacterial culture [26]. However, recent comprehensive bacterial analysis using the gene for $16 \mathrm{~S}$ ribosomal RNA (rRNA) have been successful in detecting bacteria that are difficult to cultivate [27]. NGS has become a popular means of examining a large number and volume of samples [28]. In this study, the association between periodontitis and atherosclerosis in the context of the microbiome in the oral cavity and atherosclerotic plaque was investigated by NGS.

\section{Results}

General conditions of patients were evaluated based on age, disease history, body mass index, blood pressure, C-reactive protein, cholesterol, and HbA1c (Table 1). There were no significant differences between both groups in terms of age, sex, other disease such as diabetes, and markers of inflammation and cholesterol. We evaluated the periodontal condition for each patient group from oral examination and plasma IgG antibody titer test (Table 2). In the control group, the ratio of deep pocket probing depth (PPD) $(>4 \mathrm{~mm}$ ) was $2.2 \pm 2.1 \%$. The ratio of PPD in the periodontitis group $(30.1 \pm 6.4 \%)$ was significantly higher. Serum IgG antibody titer was significantly higher in those with periodontitis that in control group for Aggregatibacter actinomycetemcomitans (Aa) Y4, Aa ATCC29523, Aa SUNY67, Capnocytophaga ochracea (Co) S3, and Pg FDC381. 
The microbiome pattern of tongue surface was relatively similar between control samples and periodontitis samples (Fig. 1A). Among them, the ratio of Filifactor was significantly higher in periodontitis patients than in the respective controls (Fig. 1B).

The microbiome pattern of gingival pockets were notably different between the control and periodontitis samples (Fig. 2A). The ratio of Rothia and Neisseria were lower in periodontitis than in control samples. Conversely, the ratios of Fusobacterium and Filifactor were higher in periodontitis than in control samples (Fig. 2A, B). The ratio of Desulfobulbus was significantly higher in periodontitis samples than in controls (Fig. 2B).

The majority of the bacteria found in the atheromatous plaques microbiome belonged to the soil bacterial families Burkholderiales, Bacillale, and Rhizobiales. Their ratios were similar between periodontitis and control patients (Fig. 3). The ratio of Sphingomonadales was higher in periodontitis samples than in control samples.

Three gingival pocket bacteria from periodontitis ( $\mathrm{P} 2, \mathrm{P} 3, \mathrm{P} 4$; red) and one control sample ( $\mathrm{H} 4$; blue) were positioned in the center right side of the PCA graph (separated by a red solid circle), three gingival pocket bacteria from control samples $(\mathrm{H} 1, \mathrm{H} 2, \mathrm{H} 3)$ and one gingival bacteria from periodontitis (P4) were located into the center of the plot (separated by a blue solid circle), respectively (Fig. 4). The two circular locations were sufficiently separated. Tongue surface bacteria from periodontitis samples were located in the center left side of the panel (P1, P2, P3, P4) (separated by a red dotted circle). This position was slightly toward to the right side than that of the control samples $(\mathrm{H} 1, \mathrm{H} 2, \mathrm{H} 3, \mathrm{H} 4)$ (separated by a blue dotted circle). These two circular locations were comparatively closer. The bacteria in atheromatous plaques were located towards the lower middle region of the plot (separated by a green solid circle), and control and periodontitis atheroma samples could not be clearly distinguished between them. The atheromatous plaques bacteria were located far away from the oral samples (tongue surface and gingival pockets).

Similar to the PCA results, bacteria from oral samples (tongue surface and gingival pocket) and atheromatous plaques were completely different (Fig. 5). However, $75 \%$ of atheromatous plaques bacteria from periodontitis were located at the lower part in the cluster and $75 \%$ of atheromatous plaques bacteria from controls were located at the upper part of the periodontitis samples.

We evaluated the correlation of the microbiome in atheromatous plaques between the control and periodontitis group. In both groups, the major bacteria of the network were Agrobacterium, Delftia, and Rhizobium. However, the network around Cutibacterium was different between control and periodontitis samples (Fig. 6).

\section{Discussion}


In this research, we performed a pilot study regarding a comprehensive microbiome analysis of the internal carotid artery stenosis in patients affected with and without periodontitis. We harvested bacteria samples from the tongue surface, gingival pockets, and atheromatous plaques from each patient and performed NGS analysis. The microbiome in the oral cavity was higher for the periodontal bacterial pathogens Fusobacterium and Filifactor in the periodontitis group compared to the control group. In particular, the ratio of Filifactor on the tongue surface was significantly higher in the periodontitis group in comparison to the control group. Conversely, the ratio of Rothia and Neisseria in gingival pockets, which are constituents of the normal bacterial flora in a healthy oral cavity, was lower in the periodontitis group than in the control group [29,30]. Thus, remarkably, the ratio of normal bacteria in gingival pockets and on the tongue surface decreased, while the ratio of pathogenic bacteria increased.

As pathogenic factors for periodontitis, Red complex species ( $P g, T$. denticola, and T. forsythia) have been the focus of functional investigations [31]. Although there is no doubt regarding their relationship to periodontitis development, the microbiome does not contain just the pathogenic bacteria, but includes a mixture of various and diverse species of bacteria, to comprise the total population ultimately affecting the development of this disease [32]. It has been suggested that 17 novel bacteria including Filifactor alosis probably induce periodontitis, even though these bacteria were not previously thought to be periodontitis-specific pathogenic bacteria [33].

The normal bacterial flora in the oral cavity, which was previously disregarded as insignificant, is actually very crucial for periodontitis development or progression. In general, pathogenic bacteria, such as $\mathrm{Pg}$, configure the microbiome with the normal bacteria flora [34]. If the balance of pathogenic and normal bacteria in the microbiome is lost for some reason, the microbiome increases its pathogenicity and induces the disease. Therefore, a comprehensive microbiome analysis is necessary to investigate normal as well as pathogenic bacteria composition.

To investigate the possibility that periodontal bacteria might contribute to atheromatous plaques formation directly on the vascular wall by hematogenous spread, NGS analysis was done using the atheromatous plaque samples. Previous reports established that $P g$ induces the expression of vascular cell adhesion molecule 1 from vascular endothelial cells, and promotes thrombus formation by macrophage invasion into blood vessels, resulting in platelet aggregation $[35,36]$. Another report demonstrated that $P g$ infection accelerates the progression of atherosclerosis in a heterozygous apolipoprotein E-deficient murine model [23]. In the present study, oral bacteria were barely detectable in atheromatous plaques, regardless of the presence or absence of periodontitis. The patterns of the microbiome in atheromatous plaques were entirely different on the tongue surface and in gingival pockets. A prior study reported the detection of some oral bacteria in the atheromatous plaques [37]. However, other authors reported that $P g$ infection in an animal model induced atheromatous plaque formation, although this bacterium was actually not detected in the atheromatous plaques [38]. Our data enables us to conclude that it is unlikely that the oral bacteria spread hematogenously and directly induce the formation of atheromatous plaque on the aortic wall. 
The co-occurrence analysis of the microbiome in atheromatous plaques revealed that the most significant bacteria were Agrobacterium, Delftia, and Rhizobium, which constituted the network in both groups. Although these are soil bacteria, they were also previously detected in atheromatous plaques [39]. Another significant bacterium, Cutibacterium, configured the network in the control group. The relationship with Cutibacterium was different among the control and periodontitis groups. This bacterium is categorized as a normal bacterium present on the skin and in the gut, although it was also detected in atheromatous plaques [40]. Cutibacterium reportedly can cause sarcoidosis, sepsis, and infective endocarditis, and heat-killed Cutibacterium render mice very susceptible to lipopolysaccharide (LPS) toxicity. Cutibacterium also promotes the production of cytokines, such as interleukin-12, interferongamma, and Toll-like receptor 4 [41]. Presently, it is conceivable that LPS produced by periodontal bacteria activated Cutibacterium in the blood vessels, which then form the atheromatous plaques. In this scenario, the difference of the network in atheromatous plaques between the control and periodontitis samples might be caused by LPS that is spread hematogenously, as well as by the chronic inflammatory effect. Recently, it was reported that the production of trimethylamine-N-oxide, which promotes atherosclerosis, depends upon the metabolism of the intestinal microbiome [42]. Some previous studies and the present data indicate that the loss of microbiome balance in the human body affects the development of atherosclerosis. Periodontitis has a great effect on the microbiome configuration in the oral cavity and promotes the formation of various metabolic products. However, this detailed mechanism of atherosclerosis development remains largely unknown. In a further study, we intend to investigate the relationship between periodontitis and atherosclerosis.

\section{Conclusion}

The ratio of oral bacteria in atheromatous plaques was remarkably low, and the microbiome pattern in the atheromatous plaques was entirely different from that found in the oral microbiome. In other words, oral bacteria did not directly induce the atheromatous plaque configuration. However, the microbiome pattern and the correlation of the microbiome in atheromatous plaques were different between the controls and periodontitis samples. Thus, metabolic products of the microbiome, or the host's inflammatory response, might indirectly affect the atheromatous plaque configuration.

\section{Materials And Methods}

\section{Ethics statement}

This study was approved by the ethics committee of Okayama University Graduate School of Medicine, Dentistry, and Pharmaceutical Sciences and Okayama University Hospital (Authorization Number: 1603059) and Brain Attack Center Ota Memorial Hospital (Authorization Number: 121). All enrolled patients provided written informed consent for the use of their resected tissue and oral samples. 


\section{Participants}

The study focused on 12 patients who visited Brain Attack Center Ota Memorial Hospital between April 2016 and March 2018, and who were diagnosed with internal carotid artery stenosis. The patients were $\geq$ 40 years of age, underwent carotid endarterectomy, had more than ten teeth, and consented to participate.

\section{Samples}

We harvested atheromatous plaques from the carotid artery walls extracted during carotid endarterectomy. Bacteria in the gingival pocket were collected using absorbent paper points (United Dental Manufactures Inc., Johnson City, TN, USA). The three paper points were inserted into patients' gingival pockets and were harvested after one minute. Each item was put them into an Eppendorf tube containing PBS. Later, bacteria from the tongue surface were collected using forensic swabs (Sarstedt AG \& Co. Nümbrecht, Germany) by wiping the dorsum portion of each tongue several times. Each swab was placed in a tube and immediately stored at $-80^{\circ} \mathrm{C}$ until DNA purification. Blood samples were collected from each patient and serum was prepared as previously described [43].

\section{Oral examination}

Periodontal examinations were performed to evaluate the average pocket probing depth (PPD) and rate of bleeding on probing (BOP) for each teeth of each patient. The patients were then divided into three groups according to the Japanese Society for Periodontology Clinical Practice Guideline for the Periodontal Treatment [44]. Briefly, patients with $<5 \%$ of deep PPD ( $>4 \mathrm{~mm})$ were defined as periodontally healthy (control, $n=4 ; \mathrm{H} 1-\mathrm{H} 4$ ), patients $(n=4)$ with $6-23 \%$ deep PPD were defined as mild periodontitis and were excluded from further analysis, and patients with $>24 \%$ of deep PPD were designated as severe periodontitis (periodontitis, $n=4 ; P 1-P 4)$.

\section{DNA purification}

APs were extensively minced using a scalpel and suspended in phosphate buffered saline (PBS). The collected material from paper points and swabs were resuspended using PBS. One milliliter of each resuspended bacterial sample was transferred to $2 \mathrm{ml}$ Lysing Matrix B tubes (MP Biomedicals, Santa Ana, CA, USA) containing $0.1 \mathrm{~mm}$ silica beads and $500 \mu \mathrm{l}$ ATL buffer (Qiagen, Hilden, Germany). The contents of each tube were homogenized using FastPrep 24 (MP Biomedicals) for $45 \mathrm{~s}$ at $6.5 \mathrm{~m} / \mathrm{s}$. Bacterial DNA was extracted using the QIAamp DNA Microbiome Kit (Qiagen) according to the manufacturer's instructions. The quality and quantity of the DNA were verified using the NanoDrop 2000 
spectrophotometer (Thermo Fisher Scientific, Wilmington, DE, USA) and the PicoGreen dsDNA assay kit (Life Technologies, Grand Island, NY, USA).

\section{Library preparation, sequencing, and analysis of 16S rRNA}

The V3 and V4 regions of the 16S rRNA was amplified using the forward primer 5'TCGTCGGCAGCGTCAGATGTGTATAAGAGACAGCCTACGGGNGGCWGCAG, and the reverse primer 5'GTCTCGTGGGCTCGGAGATGTGTATAAGAGACAGGACTACHVGGGTATCTAATCC. Thermal cycling conditions were $98^{\circ} \mathrm{C}$ for $3 \mathrm{~min} ; 25$ cycles of $98^{\circ} \mathrm{C}$ for $30 \mathrm{~s}, 55^{\circ} \mathrm{C}$ for $30 \mathrm{~s}$, and $72^{\circ} \mathrm{C}$ for $30 \mathrm{~s}$; and a final extension at $72^{\circ} \mathrm{C}$ for 5 min. After PCR clean-up step using AMpure XP Beads (Beckman Coulter, Inc., Brea, CA, USA), a second PCR was performed to add sequencing adapters and dual-index barcodes to the amplicon target to distinguish amplicons from each sample using the same reaction conditions with only eight cycles instead. After the PCR clean-up step, the quality and quantity of the amplicon were verified using KAPA library quantification kit (KAPA Biosystems Inc., Wilmington, MA, USA). Aliquots $(5 \mu \mathrm{L})$ of the diluted amplicon from each library were combined to form pooling libraries. Six PM of pooling libraries with PhiX was sequenced using the MiSeq ${ }^{\circledR}$ system (Illumina Inc., San Diego, CA, USA). The obtained sequence was analyzed using the CLC Microbial Genomics Module of the CLC Genomics Workbench (CLC bio, Aarhus, Denmark). Briefly, we extracted 5911 bacteria having sequences that passed both those being retained and operational taxonomic units (OTUs) selected based on a $97 \%$ similarity with a minimum of 10 reads representing each OTU [45]. Principal component analysis (PCA) and clustering analysis were performed using $\mathrm{R}$ statistical software [39]. We also performed co-occurrence analysis for the 13 highly detected operational taxonomic units from control and periodontitis samples using the Quantitative Insights Into Microbial Ecology approach [46].

\section{Plasma IgG antibody titer test against periodontal bacteria}

Plasma IgG antibody titer against periodontal bacteria was determined as described previously [22, 43, 47,48]. Bacterial antigens used were sonicated preparations of Aggregatibacter actinomycetemcomitans (Aa) Y4, Aa ATC29523, Aa SUNY67, Eichenerra corrodens (Ec) FDC1073, Fusobacterium nucleatum (Fn) ATCC25586, Prevotella intermedia (Pi) ATCC25611, P. nigrescens (Pn) ATCC33563, Capnocytophaga ochracea (Co) S3, Porphyromonas gingivalis (Pg) FDC381, Pg SU63, Treponema denticola (Td) ATCC35405, and Tannerella forsythia (Tf)ATCC43037. The sera from five healthy participants without periodontitis (24-29 years of age) were pooled and used to calibrate the analyses. Standard titration curves were prepared using serial dilutions of this pooled control serum. The absorbance of each sample after reaction was defined as an ELISA unit (EU), with 100 EUs corresponding to a 1:3,200 dilution of the calibrator sample [43]. According to the formula for clinical use, the mean \pm 2 standard deviations of the controls, based on the reported dataset of IgG titers to individual pathogens among five healthy individuals, was defined as the standard value of 1 . 


\section{Statistical analysis}

The statistical analysis was performed using the Mann-Whitney UTest. A P-value of 0.05 was considered significant and was determined using SPSS Ver. 23 (SPSS Inc., Chicago, IL, USA) for all the experimental results.

\section{Abbreviations}

NGS: Next-generation sequencing; LDL: low-density lipoprotein; Pg: Porphyromonas gingivalis; Aa: Aggregatibacter actinomycetemcomitans; Co: Capnocytophaga ochracea; rRNA: ribosomal RNA; PPD: pocket probing depth; BOP: rate of bleeding on probing; PCA: principal component analysis

\section{Declarations}

\section{Acknowledgments}

We thank all the staff in the Department of Biobank, Okayama University Hospital for helping our research. We also wish to acknowledge all the department staff at the Department of PathophysiologyPeriodontal Science of Okayama University Graduate School of Medicine, Dentistry and Pharmaceutical Sciences for supporting this study.

\section{Authors' contributions}

DI performed samples collection, conducted the experimental works, interpreted the results, and wrote the draft manuscript. KY supervised all experiments and edited the manuscript. KM performed the oral examination and sample collection. MT performed co-occurrence analysis. SO, MS, YS operated patients and collected samples. MT and ST performed NGS analysis. ZA performed English editing for the manuscript. TK, KO, TY gave professional advises. ST was responsible for the study, supervised and edited the manuscript. All authors read and approved the final manuscript.

\section{Funding}

This study was funded by Okayama University Grant.

\section{Availability of data and materials}

All datasets present in the research article were submitted as supplementary data. 


\section{Ethics approval and consent to participate}

This study was approved by the ethics committee of Okayama University Graduate School of Medicine, Dentistry, and Pharmaceutical Sciences and Okayama University Hospital (Authorization Number: 1603059) and Brain Attack Center Ota Memorial Hospital (Authorization Number: 121).

\section{Consent for publication}

We obtained contents for publication from participants.

\section{Competing interests}

The authors declare that they have no competing interests.

\section{Author details}

1Department of Pathophysiology-Periodontal Science, Okayama University Graduate School of Medicine, Dentistry, and Pharmaceutical Sciences. 2-5-1 Shikata-cho, Kita-ku, Okayama 700-8525, Japan,

${ }^{2}$ Department of Periodontics and Endodontics, Okayama University Hospital. 2-5-1 Shikata-cho, Kita-ku, Okayama 700-8525, Japan, ${ }^{3}$ Taniguchi Dental Clinic, Oral Bacterial Flora Analysis Center, 1-9-14, Tokiwacho, Takamatsu, Kagawa, 760-0054, Japan, ${ }^{4}$ Department of Biobank, Okayama University Graduate School of Medicine, Dentistry and Pharmaceutical Sciences. 2-5-1 Shikata-cho, Kita-ku, Okayama 7008525, Japan, ${ }^{5}$ Department of Neurology, Brain Attack Center Ota Memorial Hospital, 3-6-28, Okinogamicho, Fukuyama, Hiroshima, 720-0825, Japan

\section{References}

1. Maddi A, Scannapieco FA. Oral biofilms, oral and periodontal infections, and systemic disease. Am J Dent. 2013;26(5): 249-254. PubMed PMID: 24479275.

2. Lalla E, Papapanou PN. Diabetes mellitus and periodontitis: a tale of two common interrelated diseases. Nat Rev Endocrinol. 2011;7(12): 738-748. Epub 2011/06/28. doi: 10.1038/nrendo.2011.106.

3. Senba T, Kobayashi Y, Inoue K, Kaneto C, Inoue M, Toyokawa S, et al. The association between selfreported periodontitis and coronary heart disease-from MY Health Up Study--. J Occup Health. 2008;50(3): 283-287. Epub 2008/04/15. 
4. Lafon A, Pereira B, Dufour T, Rigouby V, Giroud M, Béjot Y, et al. Periodontal disease and stroke: a meta-analysis of cohort studies. Eur J Neurol. 2014;21(9): 1155-1161, e66-7. Epub 2014/04/08. doi: 10.1111/ene. 12415.

5. Isoshima D, Yamashiro K, Matsunaga K, Shinobe M, Nakanishi N, Nakanishi I, et al. Assessment of pathogenesis of infective endocarditis by plasma lgG antibody titer test against periodontal bacteria. Clin Case Rep. 2017;5(10): 1580-1586. Epub 2017/08/17. doi: 10.1002/ccr3.1066.

6. Dominy SS, Lynch C, Ermini F, Benedyk M, Marczyk A, Konradi A, Nguyen M, Haditsch U, Raha D, Griffin C, Holsinger LJ, Arastu-Kapur S, Kaba S, Lee A, Ryder MI, Potempa B, Mydel P, Hellvard A, Adamowicz K, Hasturk H, Walker GD, Reynolds EC, Faull RLM, Curtis MA, Dragunow M, Potempa J. Porphyromonas gingivalis in Alzheimer's disease brains: Evidence for disease causation and treatment with small-molecule inhibitors. Sci Adv. 2019 Jan 23;5(1):eaau3333. doi: 10.1126/sciadv.aau3333. eCollection 2019 Jan.

7. Zeng XT, Deng AP, Li C, Xia LY, Niu YM, Leng WD. Periodontal disease and risk of head and neck cancer: a meta-analysis of observational studies. PLoS One. 2013;8(10): e79017. Epub 2013/10/23. doi: 10.1371/journal.pone.0079017.

8. Yoneda M, Naka S, Nakano K, Wada K, Endo H, Mawatari H, et al. Involvement of a periodontal pathogen, Porphyromonas gingivalis on the pathogenesis of non-alcoholic fatty liver disease. BMC Gastroenterol. 2012;12: 16. Epub 2012/02/16. doi: 10.1186/1471-230X-12-16. PubMed PMID: 22340817; PubMed Central

9. Sanz M, Kornman K; working group 3 of the joint EFP/AAP workshop. Periodontitis and adverse pregnancy outcomes: consensus report of the Joint EFP/AAP Workshop on Periodontitis and Systemic Diseases. J Periodontol. 2013;84(4 Suppl): S164-169. doi: 10.1902/jop.2013.1340016.

10. Ross R. Atherosclerosis--an inflammatory disease. N Engl J Med. 1999;340(2): 115-126. doi: 10.1056/NEJM199901143400207.

11. Vinchi F, Muckenthaler MU, Da Silva MC, Balla G, Balla J, Jeney V. Atherogenesis and iron: from epidemiology to cellular level. Front Pharmacol. 2014 May 5;5:94. doi: 10.3389/fphar.2014.00094. eCollection 2014. Review.

12. Bahekar AA, Singh S, Saha S, Molnar J, Arora R. The prevalence and incidence of coronary heart disease is significantly increased in periodontitis: a meta-analysis. Am Heart J. 2007;154(5): 830837. Epub 2007/08/20. doi: 10.1016/j.ahj.2007.06.037.

13. Ishihara K, Nabuchi A, Ito R, Miyachi K, Kuramitsu HK, Okuda K. Correlation between detection rates of periodontopathic bacterial DNA in coronary stenotic artery plaque and in dental plaque samples. J Clin Microbiol. 2004;42(3): 1313-1315.

14. Okuda K, Ishihara K, Nakagawa T, Hirayama A, Inayama Y. Detection of Treponema denticola in atherosclerotic lesions. J Clin Microbiol. 2001;39(3): 1114-1117. doi: 10.1128/JCM.39.3.11141117.2001.

15. Lockhart PB, Bolger AF, Papapanou PN, Osinbowale O, Trevisan M, Levison ME, et al. Periodontal disease and atherosclerotic vascular disease: does the evidence support an independent 
association?: a scientific statement from the American Heart Association. Circulation. 2012;125(20): 2520-2544. Epub 2012/04/18. doi: 10.1161/CIR.0b013e31825719f3.

16. Hujoel PP, Drangsholt M, Spiekerman C, DeRouen TA. Periodontal disease and coronary heart disease risk. JAMA. 2000;284(11): 1406-1410.

17. Peacock ME, Carson RE. Frequency of self-reported medical conditions in periodontal patients. J Periodontol. 1995;66(11): 1004-1007. doi: 10.1902/jop.1995.66.11.1004.

18. Kurihara N, Inoue Y, Iwai T, Umeda M, Huang Y, Ishikawa I. Detection and localization of periodontopathic bacteria in abdominal aortic aneurysms. Eur J Vasc Endovasc Surg. 2004;28(5): 553-558. doi: 10.1016/j.ejvs.2004.08.010.

19. Beck JD, Garcia RJ, Heiss G, Vokonas PS, Offenbacher S. Periodontal disease and cardiovascular disease. J Periodontol, 67: 1123-1137, 1996.

20. Haraszthy VI, Zambon JJ, Trevisan M, Zeid M, Genco RJ. Identification of periodontal pathogens in atheromatous plaques. J Periodontol. 2000;71:1554-1560.

21. Nakano K, Inaba H, Nomura R, Nemoto H, Takeuchi H, Yoshioka H, Toda K, Taniguchi K, Amano A, Ooshima T. Distribution of Porphyromonas gingivalis fimA genotypes in cardiovascular specimens from Japanese patients. Oral Microbiol Immunol. 2008;23:170-172.

22. Kudo C, Shin WS, Minabe M, Harai K, Kato K, Seino H, Goke E, Sasaki N, Fujino T, Kuribayashi N, Pearce YO, Taira M, Maeda H, Takashiba S. Periodontitis and Atherosclerosis Project-Tokyo and Chiba Consortiums. Analysis of the relationship between periodontal disease and atherosclerosis within a local clinical system: a cross-sectional observational pilot study. Odontology. 2015 Sep;103(3):314-321. doi: 10.1007/s10266-014-0172-3. Epub 2014 Aug 14.

23. Li L, Messas E, Batista EL, Levine RA, Amar S. Porphyromonas gingivalis infection accelerates the progression of atherosclerosis in a heterozygous apolipoprotein E-deficient murine model. Circulation. 2002;105(7): 861-867.

24. Ford PJ, Gemmell E, Timms P, Chan A, Preston FM, Seymour GJ. Anti-P. gingivalis response correlates with atherosclerosis. J Dent Res. 2007 Jan;86(1):35-40.

25. Maekawa T, Takahashi N, Tabeta K, Aoki Y, Miyashita H, Miyauchi S, Miyazawa H, Nakajima T, Yamazaki K. Chronic oral infection with Porphyromonas gingivalis accelerates atheroma formation by shifting the lipid profile. PLoS One. 2011;6(5):e20240.

26. Papapanou PN, Sellén A, Wennstr JL, Dahlén An analysis of the subgingival microflora in randomly selected subjects. Oral Microbiol Immunol. 1993;8(1): 24-29.

27. Goodrich JK, Waters JL, Poole AC, Sutter JL, Koren O, Blekhman R, et al. Human genetics shape the gut microbiome. Cell. 2014;159(4): 789-799. doi: 10.1016/j.cell.2014.09.053.

28. Buffie CG, Bucci V, Stein RR, McKenney PT, Ling L, Gobourne A, et al. Precision microbiome reconstitution restores bile acid mediated resistance to Clostridium difficile. Nature. 2015;517(7533): 205-208. Epub 2014/10/22. doi: 10.1038/nature13828.

29. Wang J, Qi J, Zhao H, He S, Zhang Y, Wei S, et al. Metagenomic sequencing reveals microbiota and its functional potential associated with periodontal disease. Sci Rep. 2013;3: 1843. doi: 
10.1038/srep01843.

30. Takeshita T, Matsuo K, Furuta M, Shibata Y, Fukami K, Shimazaki Y, et al. Distinct composition of the oral indigenous microbiota in South Korean and Japanese adults. Sci Rep. 2014;4: 6990. Epub 2014/11/11. doi: 10.1038/srep06990.

31. Socransky SS, Smith C, Haffajee AD. Subgingival microbial profiles in refractory periodontal disease. J Clin Periodontol. 2002;29(3): 260-268.

32. Page RC, Kornman KS. The pathogenesis of human periodontitis: an introduction. Periodontol 2000. 1997;14: 9-11.

33. Takeshita T, Nakano Y, Kumagai T, Yasui M, Kamio N, Shibata Y, et al. The ecological proportion of indigenous bacterial populations in saliva is correlated with oral health status. ISME J. 2009;3(1): 6578. Epub 2008/10/02. doi: 10.1038/ismej.2008.91.

34. Pérez-Chaparro PJ, Gonçalves C, Figueiredo LC, Faveri M, Lobão E, Tamashiro N, et al. Newly identified pathogens associated with periodontitis: a systematic review. J Dent Res. 2014;93(9): 846858. Epub 2014/07/29. doi: 10.1177/0022034514542468

35. Khlgatian M, Nassar H, Chou HH, Gibson FC, Genco CA. Fimbria-dependent activation of cell adhesion molecule expression in Porphyromonas gingivalis-infected endothelial cells. Infect Immun. 2002;70(1): 257-267.

36. Imamura T, Travis J, Potempa J. The biphasic virulence activities of gingipains: activation and inactivation of host proteins. Curr Protein Pept Sci. 2003;4(6): 443-450.

37. Toyofuku T, Inoue Y, Kurihara N, Kudo T, Jibiki M, Sugano N, et al. Differential detection rate of periodontopathic bacteria in atherosclerosis. Surg Today. 2011;41(10): 1395-1400. Epub 2011/09/16. doi: 10.1007/s00595-010-4496-5.

38. Jain A, Batista EL, Serhan C, Stahl GL, Van Dyke TE. Role for periodontitis in the progression of lipid deposition in an animal model. Infect Immun. 2003;71(10): 6012-6018. PubMed PMID: 14500522; PubMed Central PMCID: PMCPMC201045.

39. Ziganshina EE, Sharifullina DM, Lozhkin AP, Khayrullin RN, Ignatyev IM, Ziganshin AM. Bacterial Communities Associated with Atherosclerotic Plaques from Russian Individuals with Atherosclerosis. PLoS One. 2016;11(10): e0164836. Epub 2016/10/13. doi: 10.1371/journal.pone.0164836.

40. Koren O, Spor A, Felin J, Fåk, F, Stombaugh J, Tremaroli V, et al. Human oral, gut, and plaque microbiota in patients with atherosclerosis. Proc Natl Acad Sci U S A. 2011;108 Suppl 1: 4592-4598. Epub 2010/10/11. doi: 10.1073/pnas.1011383107.

41. Tsutsui H, Imamura M, Fujimoto J, Nakanishi K. The TLR4/TRIF-mediated activation of NLRP3 inflammasome underlies endotoxin-induced liver injury in mice. Gastroenterol Res Pract. 2010;2010: 641865. Epub 2010/06/16. doi: 10.1155/2010/641865.

42. Tang WH, Wang Z, Levison BS, Koeth RA, Britt EB, Fu X, et al. Intestinal microbial metabolism of phosphatidylcholine and cardiovascular risk. N Engl J Med. 2013;368(17): 1575-1584. doi: 10.1056/NEJMoa1109400. 
43. Kudo C, Naruishi K, Maeda H, Abiko Y, Hino T, Iwata M, et al. Assessment of the plasma/serum IgG test to screen for periodontitis. J Dent Res. 2012;91(12): 1190-1195. Epub 2012/09/26. doi: $10.1177 / 0022034512461796$.

44. Japanese Society of Periodontology. JSP Clinical Practice Guideline the Periodontal Treatment; 2015. http://www.perio.jp/publication/upload_file/guideline_perio_plan2015.pdf

45. Emery DC, Shoemark DK, Batstone TE, Waterfall CM, Coghill JA, Cerajewska TL, Davies M, West NX, Allen SJ. 16S rRNA Next Generation Sequencing Analysis Shows Bacteria in Alzheimer's PostMortem Brain. Front Aging Neurosci. 2017 Jun 20;9:195. doi: 10.3389/fnagi.2017.00195. eCollection 2017.

46. Shi W, Qin M, Chen F, Xia B. Supragingival microbial profiles of permanent and deciduous teeth in children with mixed dentition. PLoS One. 2016;11(1): e0146938. Epub 2016/01/11. doi: 10.1371/journal.pone.0146938.

47. Murayama Y, Nagai A, Okamura K, Kurihara H, Nomura Y, Kokeguchi S, et al. Serum immunoglobulin G antibody to periodontal bacteria. Adv Dent Res. 1988;2(2): 339-345. doi: $10.1177 / 08959374880020022401$.

48. Sugi N, Naruishi K, Kudo C, Hisaeda-Kako A, Kono T, Maeda H, Takashiba S. Prognosis of periodontitis recurrence after intensive periodontal treatment using examination of serum IgG antibody titer against periodontal bacteria. J Clin Lab Anal. 2011;25(1):25-32.

\section{Tables}

Due to technical limitations, tables 1 and 2 are only available as a download in the supplemental files section.

\section{Figures}



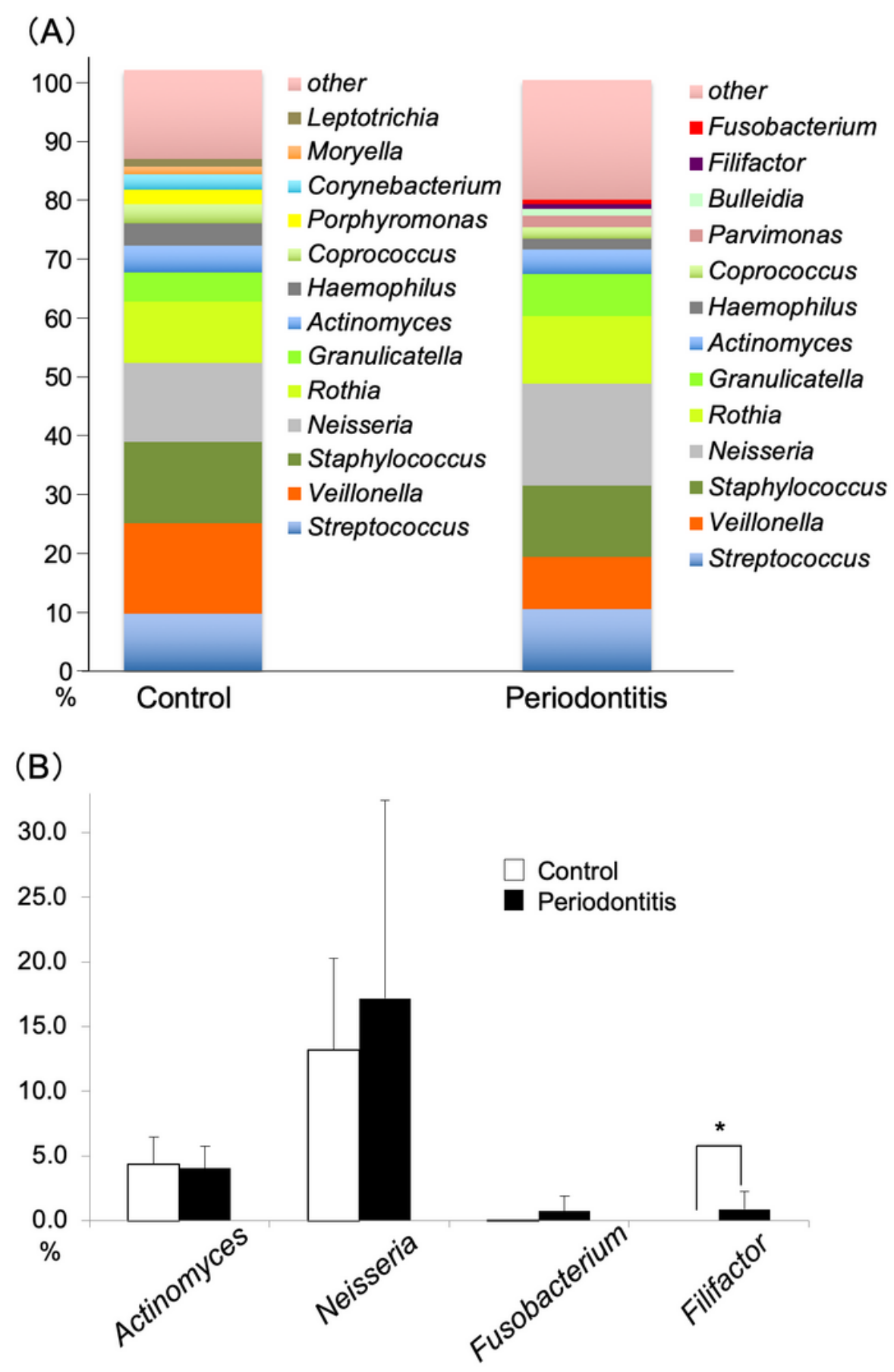

Figure 1

Figure 1

Characterization of microbiome on the tongue surface The average ratio of the bacteria on the tongue surface from control and periodontitis patient samples is presented. (A) Bacterial genera are indicated.

(B) Thirteen bacterial species were highly detected by NGS analysis. * indicates $P<0.05$; Mann-Whitney $U$ Test. 

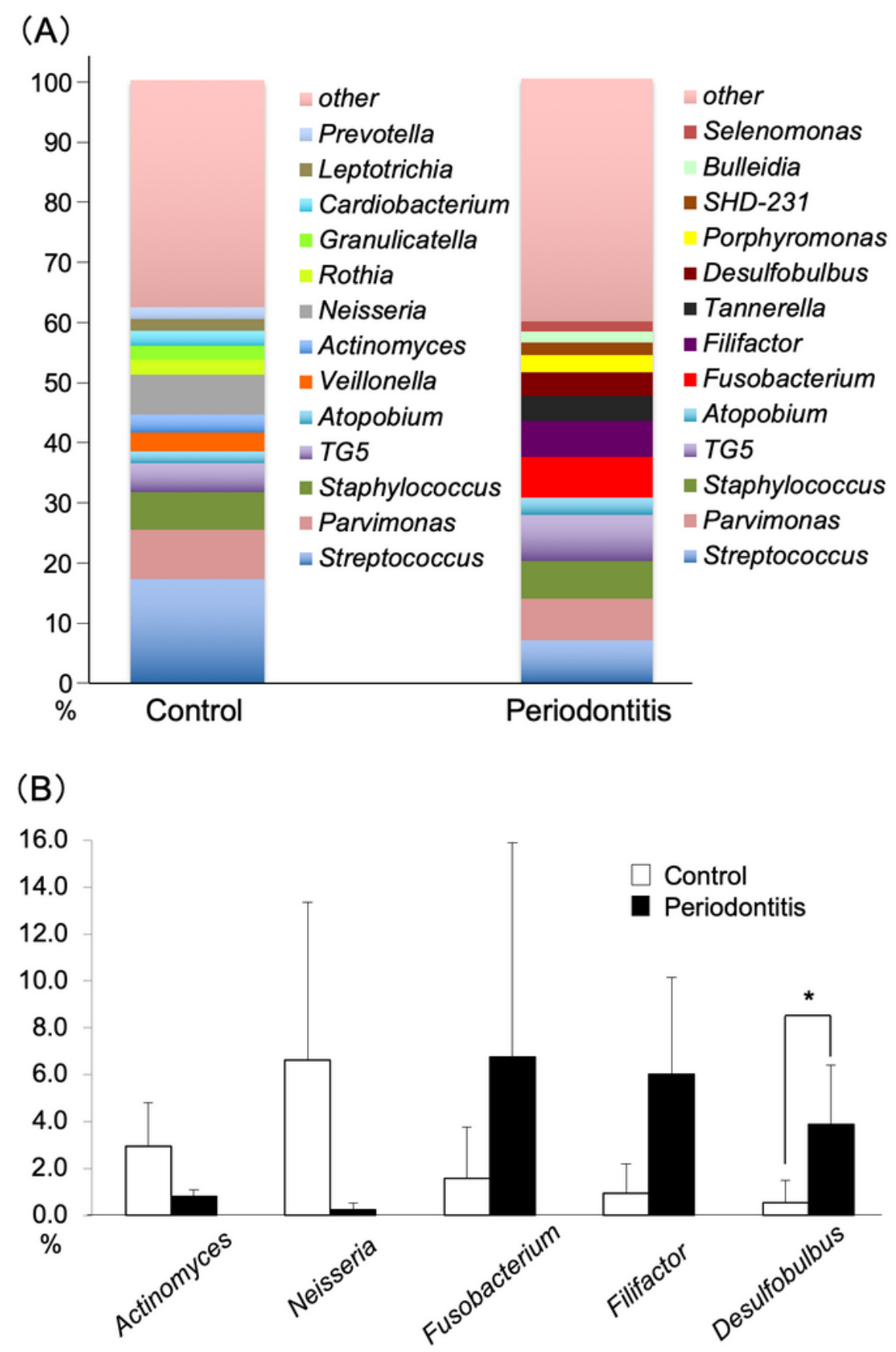

Figure 2

Figure 2

Characterization of microbiome in gingival pockets The average ratios of the bacteria in gingival pockets from control and periodontitis samples are shown. (A) Bacterial genera are indicated. (B) Thirteen bacteria that were highly detected from the NGS analysis. * indicates $\mathrm{P}<0.05$; Mann-Whitney U Test. 


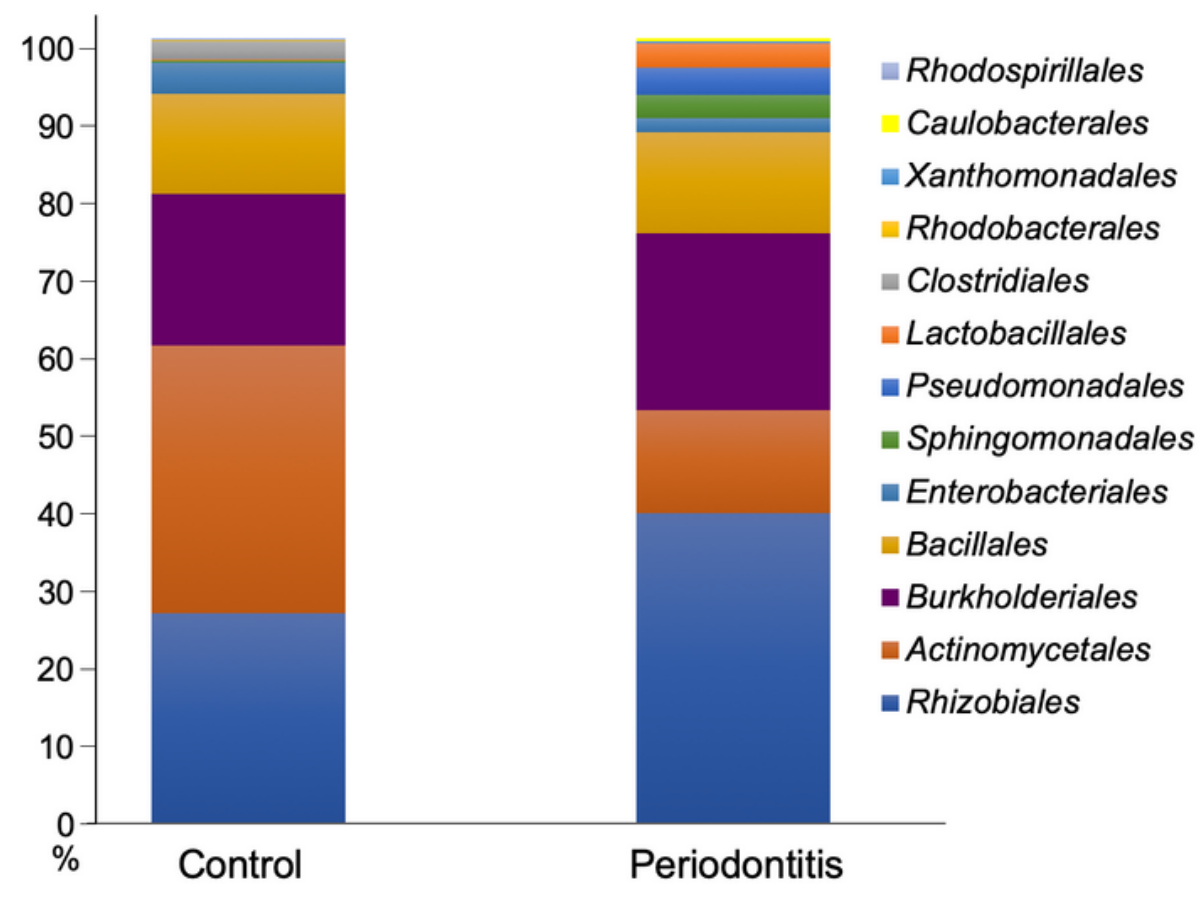

Figure 3

\section{Figure 3}

Characterization of microbiome in atheromatous plaques The average ratio of the bacteria in gingival pocket from our patients (control and periodontitis samples) is depicted and the order of the bacteria is shown. Thirteen bacteria were highly expressed from the NGS analysis. 


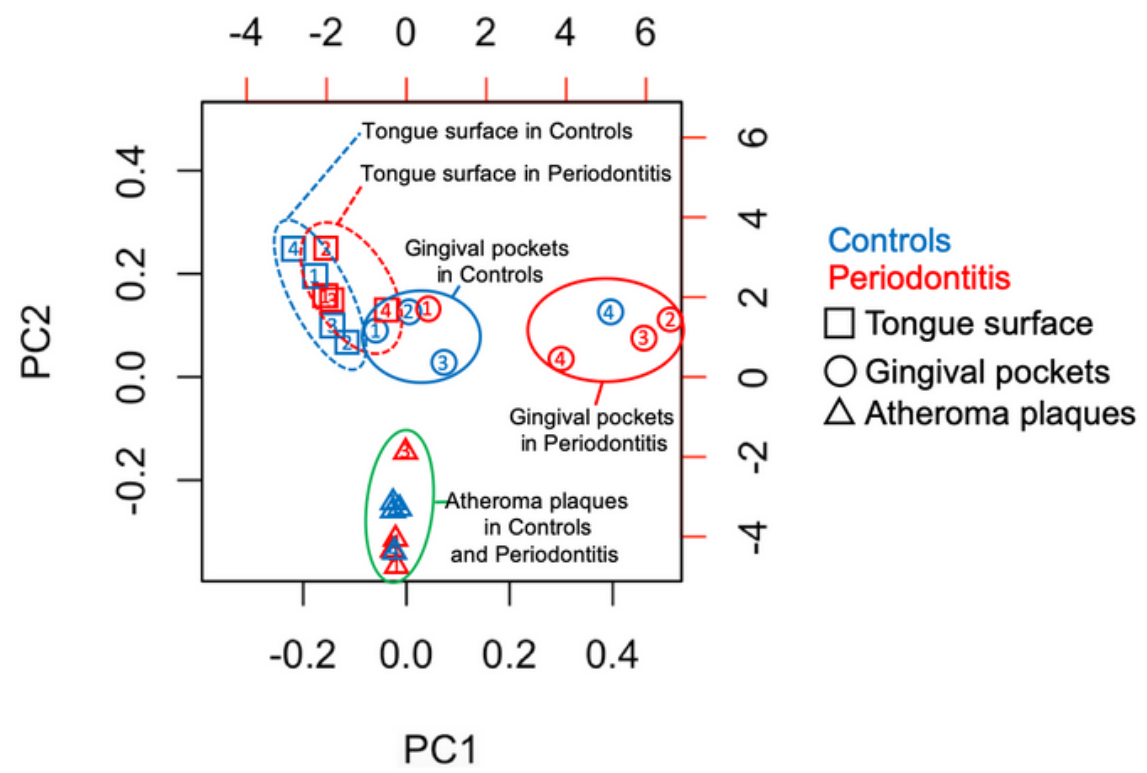

Figure 4

Figure 4

Comparison of PCA results between control and periodontitis samples The PCA results from each sample are identified tagged as follows: tongue surface: $\square$; gingival pocket: $\bullet$; atheromatous plaques: $\Delta$; controls: blue color; periodontitis: red color. Blue solid circle: three gingival pocket bacteria from control samples and one gingival pocket from periodontitis. Red solid circle: three periodontitis gingival bacteria pocket and one healthy gingival pocket sample. Red dotted circle: four tongue surface from periodontitis 
samples. Blue dotted circle: four tongue surface from control samples. Green solid circle: four atheroma plaque samples from controls and four atheroma samples from periodontitis.

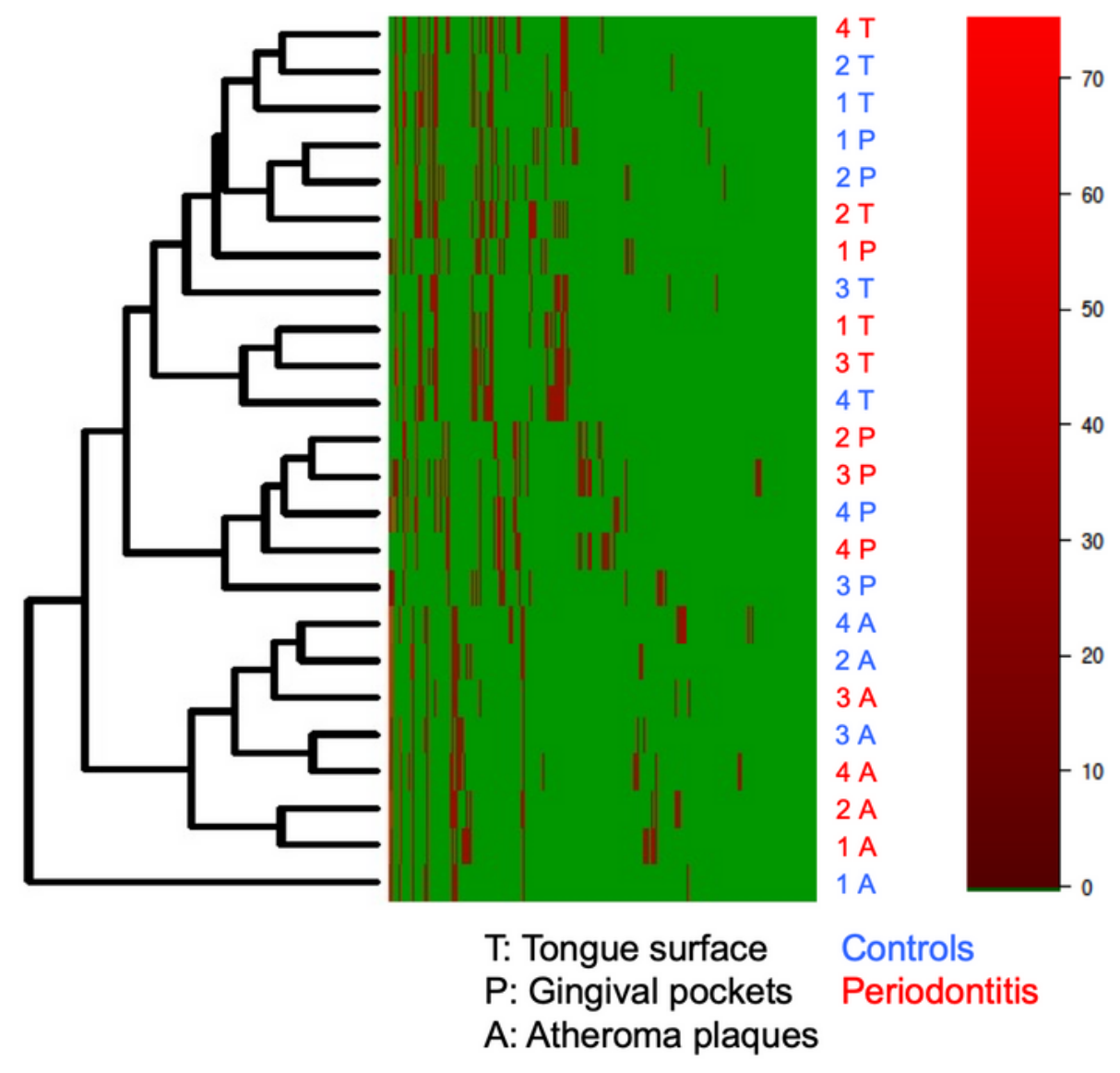

Figure 5

Figure 5

Comparison of clustering analysis results between control and periodontitis samples The clustering analysis results from each sample were tagged as follows: T: tongue surface; $P$ : gingival pocket; $A$ : atheromatous plaques. Blue text represents control samples and red text specifies periodontitis samples 

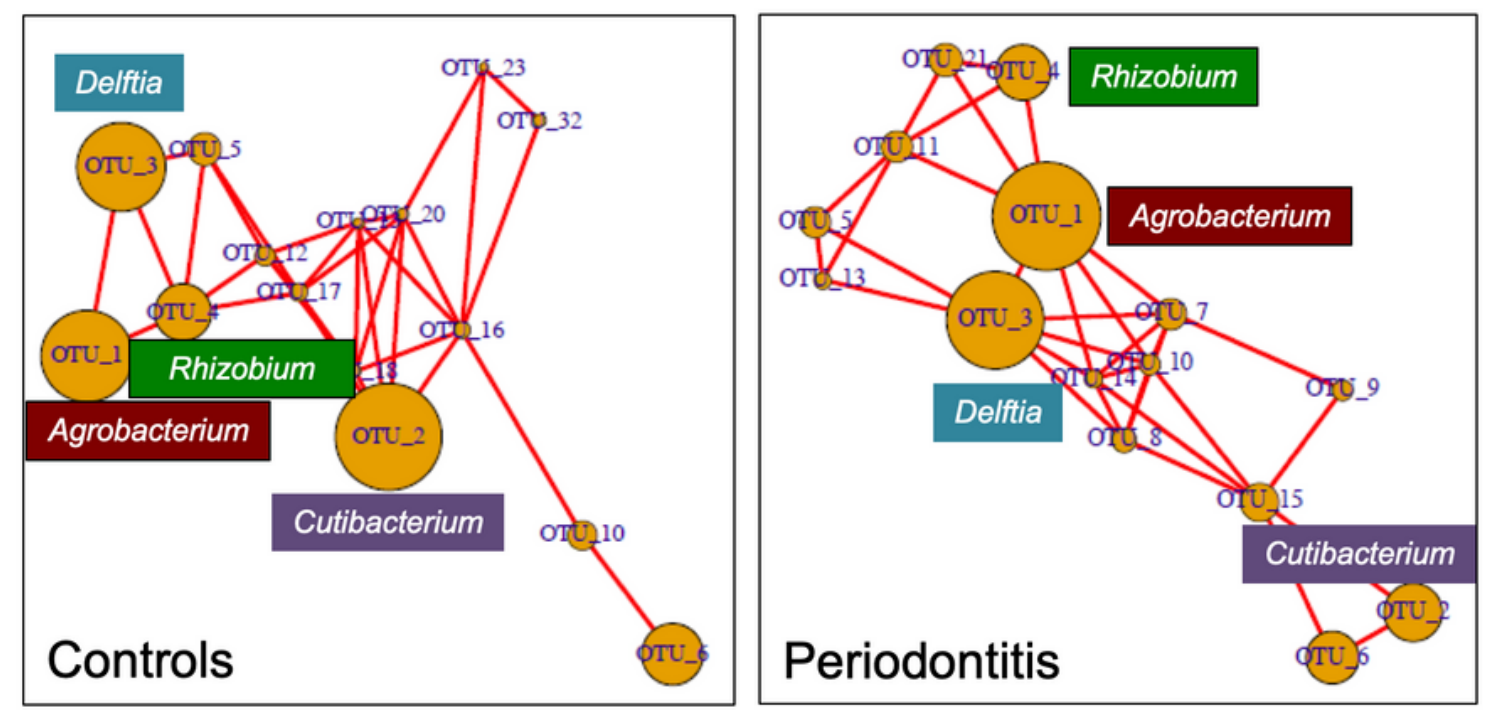

OTU: Operational Taxonomic unit

OTU_1: Agrobacterium

OTU_2: Cutibacterium

OTU_3: Delftia

OTU_4: Rhizobium

Figure 6

\section{Figure 6}

Co-occurrence analysis of microbiome in atheromatous plaques Co-occurrence analysis for the 13 highly detected operational taxonomic units (OTUs) from control and periodontitis samples using the Quantitative Insights Into Microbial Ecology approach. correlation coefficient > 0.4. Abbreviations are: OTU_1: Agrobacterium®OTU_2: Cutibacterium®OTU_3: Delfti囚and OTU_4: Rhizobium 


\section{Supplementary Files}

This is a list of supplementary files associated with this preprint. Click to download.

- supplement1.xlsx

- supplement2.png

- supplement3.xlsx

- supplement4.xlsx

- supplement5.xlsx

- supplement6.xlsx

- supplement7.xIsx

- supplement8.png

- supplement9.xlsx 